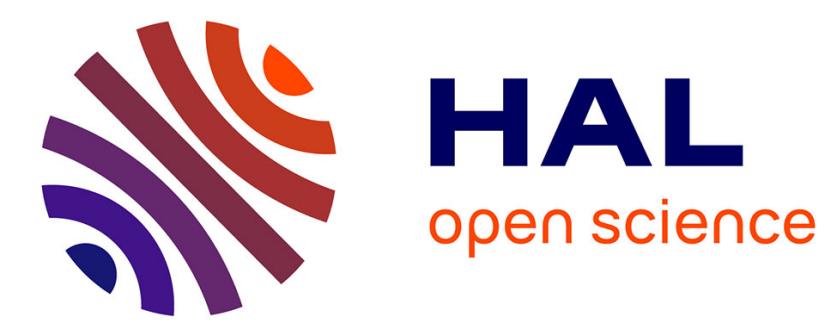

\title{
Le droit français des religions au miroir des textes
}

\author{
Françoise Curtit
}

\section{To cite this version:}

Françoise Curtit. Le droit français des religions au miroir des textes. Droit et religion en Europe. Études en l'honneur de Francis Messner, Presses universitaires de Strasbourg, pp.63-72, 2014, 978-286820-564-3. 10.4000/books.pus.9441 . halshs-01656617

\section{HAL Id: halshs-01656617 https://shs.hal.science/halshs-01656617}

Submitted on 5 Dec 2017

HAL is a multi-disciplinary open access archive for the deposit and dissemination of scientific research documents, whether they are published or not. The documents may come from teaching and research institutions in France or abroad, or from public or private research centers.
L'archive ouverte pluridisciplinaire HAL, est destinée au dépôt et à la diffusion de documents scientifiques de niveau recherche, publiés ou non, émanant des établissements d'enseignement et de recherche français ou étrangers, des laboratoires publics ou privés. 


\title{
Le droit français des religions au miroir des textes
}

\author{
Françoise CURTIT \\ (DRES, CNRS/Université de Strasbourg)
}

L'émergence depuis quelques décennies de travaux consacrés au droit des religions en France a suscité la publication de plusieurs ouvrages, manuels, recueils et rapports consacrés à cette discipline ${ }^{1}$. S'ils exposent les règles applicables en la matière ou tentent d'en dégager les principes constitutifs et les évolutions, très peu d'entre eux se hasardent à définir et caractériser cette branche du droit ${ }^{2}$. La juxtaposition de plusieurs régimes des cultes dans le cadre national (régime de séparation, droit alsacien-mosellan, droits des collectivités d'outre-mer), la diversification des sources jurisprudentielles et le rôle croissant des ajustements apportés par la pratique administrative rendent en effet délicates les tentatives d'appréhension d'une discipline qui s'appuie par ailleurs sur un ensemble de dispositions juridiques éparses et morcelées, souvent anciennes et parfois contradictoires. Le manque de lisibilité de ce corpus de règles a été souligné notamment par le rapport de la Commission Machelon qui affirme que le droit des cultes "constitue sans doute l'un des domaines du droit où l'objectif à valeur constitutionnelle d'accès et d'intelligibilité de la règle de droit est le plus gravement bafoué ${ }^{3}$.

II ne s'agira pas ici de définir ce qu'est ou n'est pas le droit des religions en France, mais de dégager quelques-unes de ses caractéristiques à partir de l'étude des normes législatives et réglementaires qui régulent le fait religieux sous ses divers aspects: liberté de religion individuelle et collective, exercice et expression du culte sous ses différentes formes, patrimoine religieux, organisation et financement des institutions religieuses... Le droit des religions ne se résume bien évidemment pas à la seule législation, mais cet ensemble de textes ancré historiquement demeure le socle autour duquel s'articulent jurisprudence, doctrine et pratiques.

La question de l'accessibilité de cet ensemble de textes, l'analyse à la fois quantitative et qualitative de sa structure et de son contenu et enfin sa mise en regard avec les statuts des cultes des autres États européens permettent d'en esquisser les qualités et les faiblesses au regard des évolutions du champ religieux.

\section{Un accès difficile aux sources formelles du droit des religions}

On peut estimer entre cent et deux cents le nombre de dispositions constituant le corpus des textes de droit français des religions, selon les critères retenus. II n'existait pas de compilation officielle visant l'exhaustivité ${ }^{4}$ avant qu'en 2011 le ministère de l'Intérieur édite un recueil de textes et de jurisprudence intitulé Laïcité et liberté religieuse, regroupant au sein de quatre thématiques environ cent vingt dispositions, soit « les principaux textes de notre

\footnotetext{
${ }^{1}$ V. notamment A. BOYER, Le droit des religions en France, Paris, PUF, 1993 ; J. VOLFF, Le droit des cultes, Paris, Dalloz, 2005 ; X. DELSOL, A. GARAY, E. TAWIL, Droit des cultes : personnes, activités, biens et structures, Paris, Juris associations, 2005 ; Liberté religieuse et régimes des cultes en droit français : textes, pratique administrative, jurisprudence, Paris, Cerf, nouv. éd. 2005 ; J.-P. MACHELON (dir.), Les relations des cultes avec les pouvoirs publics : travaux de la Commission de réflexion juridique. Rapport au ministre d'État, ministre de I'Intérieur et de l'Aménagement du territoire, Paris, La Documentation française, 2006 ; F. CURTIT, F. MESSNER (éd.), Droit des religions en France et en Europe : recueil de textes, Bruxelles, Bruylant, 2008 ; F. MESSNER (dir.), Dictionnaire Droit des Religions, Paris, CNRS Éd, 2011.

${ }^{2}$ V. cependant F. MESSNER, P.-H. PRÉLOT, J.-M. WOEHRLING (dir.), Traité de droit français des religions, Paris, Litec, $2^{\mathrm{e}}$ éd. 2012, $\mathrm{n}^{\circ} 35$ et $\mathrm{s}$.

3 J.-P. MACHELON (dir.), op. cit., p. 17.

${ }^{4}$ Des brochures thématiques avaient été publiées: Cultes et associations cultuelles, congrégations et collectivités religieuses, Paris, Journaux officiels, 1999 ; Face aux sectes, Paris, Journaux officiels, 2002.
} 
droit qui s'appliquent en matière religieuse », dans le souci de rendre la loi "intelligible et facilement accessible ${ }^{5}$. Si cette publication tente de remédier à la rareté des outils disponibles en la matière, le recours à une édition papier, donc rapidement obsolète et de diffusion relativement restreinte, vise cependant des spécialistes (juristes, praticiens du droit, universitaires...) plutôt qu'un large public comme semblaient l'indiquer les intentions premières du ministère. Un accès libre aux principales dispositions sur un site web aurait davantage répondu à l'exigence d'accessibilité du droit, à l'image de ce qui est proposé dans de nombreux pays européens par les services ministériels en charge de la gestion des cultes ${ }^{6}$.

L'accès thématique du site Legifrance, portail officiel du droit français géré par le Secrétariat général du gouvernement, n'offre quant à lui qu'une aide limitée en la matière, l'interrogation par le terme "religion » renvoyant d'une part à l'ensemble des normes relatives à la lutte contre les discriminations et, d'autre part, à une rubrique "culte " regroupant toutes les dispositions particulières à l'Alsace-Moselle (droit local des cultes, mais aussi de la chasse, de la protection sociale...) ainsi que les règles du régime de séparation, soit pour l'essentiel les textes relatifs aux associations, congrégations et fondations. Des renvois sont par ailleurs proposés vers la notion de "laïcité » appliquée notamment à l'enseignement public et vers les textes concernant les " ministres des cultes ». Cette navigation complexe ne permet pas d'accéder de façon claire et ordonnée à l'ensemble des normes du droit des religions et tend à identifier par ailleurs celui-ci principalement au droit des associations religieuses.

Pour saisir l'ensemble des dispositions de droit des religions, il faut pouvoir rassembler un grand nombre de textes et d'extraits de textes épars, vérifier leur actualisation et, pour les plus anciens, savoir les interpréter en fonction du contexte juridique actuel. Cette question de l'accessibilité - ou plutôt de la non-accessibilité - des textes juridiques constitue le premier obstacle à l'appréhension et à la compréhension du droit des religions et concourt à l'opacité de la discipline.

\section{Un droit morcelé}

Quelques tentatives de compilation ont été entreprises pour pallier cette difficulté d'accès aux textes. Les premières collections systématiques de textes relatifs au régime des cultes en droit français sont l'œuvre de juristes réunis par la Conférence des évêques de France ${ }^{7}$, puis de chercheurs au sein de l'équipe Société, droit et religion en Europe (SDRE) de l'Université de Strasbourg et du CNRS. Alimentée au sein de cette unité de recherche, la base de données LEGIREL $^{8}$ a pour objectif de donner accès aux textes internationaux relatifs à la liberté religieuse ainsi qu'à la législation des États membres de l'Union européenne concernant les organisations et activités religieuses et l'exercice des cultes. S'agissant de la France, elle répertorie plus de deux cent vingt dispositions législatives et réglementaires actuellement en vigueur, dont quatre-vingt-dix consacrées aux droits locaux de l'Alsace-Moselle et de l'outre-mer.

Parmi les cent trente dispositions concernant le régime général des cultes (loi 1905) qui composent le corpus examiné ici, une moitié environ est consacrée à titre principal au fait religieux, l'autre moitié étant constituée d'articles isolés de textes législatifs et réglementaires (par exemple, l'article 56 de la loi $\mathrm{n}^{\circ} 86-1067^{9}$ qui prévoit la programmation d'émissions religieuses sur France Télévisions) ou d'articles codifiés répartis dans une dizaine de codes distincts (Code de l'éducation, Code des impôts, Code de la sécurité sociale...). Cet éparpillement des normes reflète la transversalité de la discipline : le droit des religions n'est

\footnotetext{
${ }^{5}$ FRANCE. DIRECTION DES LIBERTÉS PUBLIQUES ET DES AFFAIRES JURIDIQUES, Laïcité et liberté religieuse : recueil de textes et de jurisprudence, Paris, Journaux officiels, 2011, p. 3.

${ }^{6}$ Danemark : sur le site www.retsinformation.dk; Espagne : sur le site www.mjusticia.gob.es ; Italie : sur le site www.governo.it ; Pologne : sur le site www.msw.gov.pl (consultés le 02.09.2013)

${ }^{7}$ Liberté religieuse et régimes des cultes en droit français : textes, pratique administrative, jurisprudence, Paris, Cerf, 1996 [nouv. éd. 2005 ].

${ }^{8}$ www.legirel.cnrs.fr

${ }^{9}$ Loi n $86-1067$ du 30 septembre 1986 relative à la liberté de communication.
} 
pas une construction juridique organisée et hiérarchisée, mais plutôt un réseau parcourant toutes les branches du droit, privé comme public (droit constitutionnel, droit administratif, droit fiscal, droit de l'urbanisme, droit pénal...), agrégeant règles de droit commun et règles propres aux institutions et activités cultuelles. Cette dispersion engendre d'ailleurs souvent une juxtaposition de dispositions pour réguler un même objet (dons et legs, édifices cultuels, congrégations religieuses...), ce qui accentue encore le manque de lisibilité de l'ensemble.

\section{Un droit archaïque ?}

Le socle des textes qui constituent aujourd'hui le droit français des religions est relativement ancien et l'on continue d'appliquer nombre de dispositions juridiques adoptées à la fin du $19^{\mathrm{e}}$ siècle et dans la première moitié du $20^{\mathrm{e}}$ siècle. Au $19^{\mathrm{e}}$ siècle est ainsi fixée la législation concernant les dons et legs aux établissements ecclésiastiques, la reconnaissance des congrégations, les aumôniers militaires, puis viennent les grandes lois de la III République (laïcité dans l'enseignement, neutralité des cimetières, liberté de réunion, laïcisation des funérailles...) qui demeurent pour partie encore largement en vigueur aujourd'hui. Au début du $20^{\mathrm{e}}$ siècle, des lois et décrets vont fixer le cadre juridique, toujours valide, de l'organisation des associations religieuses, de la police du culte, de la gestion des édifices, sans oublier le vote en 1905 de la loi de Séparation ${ }^{10}$, "mythe fondateur " de la laïcité française. Hormis quelques interventions dans de nouveaux domaines (enseignement privé, abattage rituel, protection sociale des ministres du culte...), les textes essentiellement réglementaires émis sous la $V^{e}$ République vont décliner les principes existants sans introduire de véritable rupture. Les années 2000 verront cependant le retour du recours à la loi comme expression d'une volonté politique, en matière de répression des mouvements sectaires (loi du 12 juin $2001^{11}$ ), ou de visibilité des signes religieux à l'école (loi du 15 mars $2004^{12}$ ) et dans l'espace public (loi du 11 octobre $2010^{13}$ ).

Si le droit actuel est très largement hérité de dispositions mises en œuvre il y a plus de cent ans, il n'est pas pour autant fossilisé. Si l'on excepte les textes régissant les cultes en Alsace-Moselle et en outre-mer, la plupart des normes en vigueur aujourd'hui ont été modifiées à de multiples reprises, une douzaine de fois par exemple s'agissant de la loi de 1905. Dans le domaine du droit des religions, on ne fait pas table rase pour construire une réglementation nouvelle, mais on adapte au fur et à mesure par petites touches et ajouts successifs la législation existante. II faut donc démentir une critique qui voudrait que le droit français des religions soit figé ${ }^{14}$, voire archaïque et poussiéreux : il a en effet montré au fil des décennies qu'il était pour le moins adaptable, sa nature essentiellement réglementaire facilitant d'ailleurs cette évolutivité.

\section{Un droit essentiellement réglementaire}

Figurent dans le corpus de textes étudié trois dispositions de valeur constitutionnelle (art. 10 de la Déclaration des droits de l'homme et du citoyen du 26 août 1789, préambule de la Constitution du 27 octobre 1946, art. $1^{\text {er }}$ de la Constitution du 4 octobre 1958) et une vingtaine de lois et ordonnances non codifiées, pour la plupart antérieures à 1942, dont une dizaine seulement est consacrée à titre principal au fait religieux. Force est de constater que depuis le milieu du $20^{\mathrm{e}}$ siècle le droit des religions est essentiellement un droit de nature réglementaire composé de décrets, arrêtés et circulaires, ces dernières étant particulièrement représentées (près d'un quart des dispositions en vigueur). II s'agit en général de circulaires qui ne se contentent pas d'interpréter une législation particulière pour

\footnotetext{
${ }^{10}$ Loi du 9 décembre 1905 concernant la séparation des Églises et de l'État.

${ }^{11}$ Loi $n^{\circ}$ 2001-504 du 12 juin 2001 tendant à renforcer la prévention et la répression des mouvements sectaires portant atteinte aux droits de l'homme et aux libertés fondamentales.

${ }_{12}^{12}$ Loi $n^{\circ}$ 2004-228 du 15 mars 2004 encadrant, en application du principe de laïcité, le port de signes ou de tenues manifestant une appartenance religieuse dans les écoles, collèges et lycées publics.

${ }^{13}$ Loi $n^{\circ}$ 2010-1192 du 11 octobre 2010 interdisant la dissimulation du visage dans l'espace public.

${ }^{14}$ R. ROUQUETTE, Cultes, laïcité et collectivités territoriales, Paris, Le Moniteur, 2007, p. 36.
} 
en faciliter l'application (par exemple, circulaire du 18 mai 2004 sur le port de signes religieux dans les établissements d'enseignement publics ${ }^{15}$ pour la mise en œuvre de la loi 15 mars 2004), plusieurs d'entre elles visant plutôt à rappeler les règles existantes et les possibilités d'adaptation administrative offertes par le droit commun sur des questions pour lesquelles la réglementation n'est pas claire (ou est mal comprise) ou qui font l'objet de tensions et de débats sur le terrain (circulaire du 2 février 2005 sur la laïcité dans les établissements de santé $^{16}$ ou circulaire du 19 février 2008 sur la police des lieux de sépulture ${ }^{17}$ ). Dans ce dernier cas, la circulaire joue en quelque sorte un rôle de "codification " du droit existant, mais aussi de promotion d'une volonté gouvernementale. C'est ainsi que depuis la fin des années 1990 une dizaine de circulaires ont été consacrées à la lutte contre les dérives sectaires (la dernière en date du 22 mars $2012^{18}$ ) : il ne s'agit pas uniquement de rappeler le droit, mais de promouvoir l'action des différents ministères en la matière, les textes s'additionnant au fil des gouvernements. Une dernière catégorie est composée par des circulaires qui constituent le principal, voire le seul support juridique pour réguler une activité donnée, en tout cas le seul support de la pratique administrative : c'est le cas par exemple de la circulaire du 20 décembre 2006 consacrée aux aumôniers d'hôpitaux ${ }^{19}$ ou de celle du 23 septembre 1967 relative aux autorisations d'absence des fonctionnaires pour fêtes religieuses ${ }^{20}$.

Le droit des religions est un droit réglementaire et donc assez facilement modifiable et adaptable, car c'est un droit relativement pragmatique : il s'agit pour l'essentiel de réguler des usages, d'administrer et de gérer des organisations et des personnels.

\section{Un droit centré sur la gestion des organisations}

Si l'on s'intéresse maintenant aux domaines d'intervention des textes recensés, et plus précisément à leur poids respectif au regard du nombre de dispositions qui leur sont consacrées, on peut classer les textes de droit français des religions en quatre grands groupes. Arrivent largement en tête (soit environ un quart du total) les textes consacrés à l'organisation des cultes dans un sens large (statut des associations et congrégations, droit fiscal, organisation des services d'aumôneries, personnel des institutions religieuses), suivent ceux relatifs à l'exercice du culte (police des cultes, lieux de culte, abattage rituel, funérailles), puis ceux concernant l'enseignement (laïcité dans l'enseignement public, aumôneries scolaires, établissements d'enseignement privés) et en dernier lieu les dispositions garantissant liberté de religion et non-discrimination.

Si l'on se réfère à ce seul corpus des textes juridiques, le droit des religions apparaît comme un droit technique visant à administrer et/ou financer des organisations, leurs bâtiments et leurs personnels. On a affaire ici à un droit des cultes plutôt qu'à un droit des pratiques religieuses, à un droit des communautés plutôt qu'à un droit des individus.

\section{Le droit français des religions face aux statuts des cultes des autres États européens}

Sur la base de dispositions constitutionnelles affirmant la liberté de religion, plusieurs pays européens ont adopté une loi-cadre sur la liberté de religion et le statut des organisations

\footnotetext{
${ }^{15}$ Circulaire du 18 mai 2004 relative à la mise en œuvre de la loi $n^{\circ} 2004-228$ du 15 mars 2004 encadrant, en application du principe de laïcité, le port de signes ou de tenues manifestant une appartenance religieuse dans les écoles, collèges et lycées publics.

${ }^{16}$ Circulaire DHOS/G n ${ }^{\circ} 2005-57$ du 2 février 2005 relative à la laïcité dans les établissements de santé.

${ }^{17}$ Circulaire NOR/INT/A/08/00038/C du 19 février 2008. Police des lieux de sépulture.

${ }^{18}$ Circulaire NOR/MENE1208599C du 22 mars 2012 relative à la prévention et la lutte contre les risques sectaires. Action de l'éducation nationale.

${ }^{19}$ Circulaire DHOS/P1/2006/538 du 20 décembre 2006 relative aux aumôniers des établissements mentionnés à l'article 2 de la loi $n^{\circ} 86-33$ du 9 janvier 1986 portant dispositions statutaires relatives à la fonction publique hospitalière.

${ }^{20}$ Circulaire FP/901 du 23 septembre 1967 relative aux autorisations d'absence pour fêtes religieuses.
} 
religieuses ${ }^{21}$. Ces lois vont d'une part définir les champs d'application de la liberté de religion et de l'exercice du culte et, d'autre part, déterminer différents niveaux de reconnaissance des cultes par l'État en établissant diverses strates de normes juridiques applicables aux communautés religieuses. D'autres États ${ }^{22}$, et parfois les mêmes, développent également un droit conventionnel par l'intermédiaire de concordats avec l'Église catholique ou d'accords avec les autres cultes, dont vont ensuite découler les réglementations relatives aux activités de ces confessions. Lois et accords vont servir de base pour l'élaboration de dispositions spécifiques en matière de financement des cultes, d'assistance spirituelle dans les établissements publics, d'enseignement de la religion, de reconnaissance des mariages religieux...

En France, la loi du 9 décembre 1905 énonce un certain nombre de principes, de règles d'organisation et de police, mais elle ne joue pas ce rôle de loi-cadre à l'origine d'un véritable régime des cultes, tout comme le principe de laïcité ne permet pas de définir à lui seul la place des organisations et activités religieuses dans la société. On ne dispose pas d'un véritable socle sur lequel reposerait l'ensemble des dispositions législatives et réglementaires régulant le fait religieux. Face au modèle vertical et intégré des législations autrichienne, espagnole, ou slovène, le droit français des religions présente une structure de réseau entrelaçant des normes sans véritable lien et hiérarchie entre elles et où les principes fondamentaux sont relativement peu développés alors que certaines questions « techniques » sont traitées par ailleurs de façon compulsive.

\section{Droit des religions et principe de liberté de religion}

$\mathrm{Si}$, dans tous les pays européens, la liberté de religion est garantie et encadrée par le droit international, et notamment par la Convention européenne des droits de l'homme (CEDH), la plupart des constitutions nationales comportent également un article consacré à la liberté de conscience et de religion et à ses manifestations individuelles et collectives ${ }^{23}$, très souvent inspiré de l'article 9 de la CEDH. Les textes constitutionnels parfois, les loiscadres le plus souvent, mentionnent en outre le principe de liberté d'organisation des communautés religieuses, voire font référence aux droits internes des confessions religieuses: le statut juridique des cultes et des activités confessionnelles va découler directement et explicitement du principe de liberté religieuse et de son libre exercice qui sont expressément mentionnés et définis dans les textes fondamentaux. En France, le principe de liberté de religion n'est pas en tant que tel énoncé dans la Constitution ${ }^{24}$, même s'il est par ailleurs clairement affirmé par la jurisprudence, sous l'effet notamment du rôle unificateur du droit international. Ce sont ce que d'aucuns considèrent comme ses composantes - les principes de liberté de conscience et de libre exercice du culte - qui sont énoncés dans les textes $^{25}$, et les sources juridiques en la matière sont finalement assez rares et laconiques. Le Conseil d'État relève dans un rapport consacré à la laïcité que « l'adhésion à une religion ou à une spiritualité ne se traduit pas seulement par l'exercice d'un culte. On ne peut donc

\footnotetext{
${ }^{21}$ Espagne : loi organique 7/1980 du 5 juillet sur la liberté religieuse ; Estonie : loi du 12 février 2002 sur les Églises et congrégations; Lettonie : loi du 7 septembre 1995 sur les organisations religieuses; Portugal : loi 16/2001 du 22 juin sur la liberté de religion ; République tchèque : loi 3/2002 du 7 janvier relative à la liberté d'expression religieuse et au statut des Églises et des communautés religieuses ; Roumanie : loi 489/2006 sur la liberté religieuse et le régime général des cultes ; Slovénie : loi du 2 février 2007 sur la liberté de religion...

${ }^{22}$ Allemagne, Autriche, Belgique, Espagne, Italie, Luxembourg...

${ }^{23}$ Par ex. art. 11 de la Constitution finlandaise du 11 juin 1999 : « Chacun dispose de la liberté de religion et de conscience. La liberté de religion et de conscience comprend le droit de confesser et de pratiquer une religion, le droit d'exprimer des convictions et le droit d'appartenir ou non à une communauté religieuse. Nul n'est tenu de prendre part, contre sa conscience, à la pratique d'une religion. »

${ }^{24}$ V. F. MESSNER, P.-H. PRÉLOT, J.-M. WOEHRLING (dir.), op. cit., $\mathrm{n}^{\circ} 1085$ et s.

${ }^{25}$ Art. 10 de la Déclaration française des droits de l'homme de 1789: "Nul ne doit être inquiété pour ses opinions, même religieuses, pourvu que leur manifestation ne trouble pas l'ordre public. »; Art. 1 de la Constitution de 1958: "La France est une République indivisible, laïque, démocratique et sociale. Elle assure l'égalité devant la loi de tous les citoyens sans distinction d'origine, de race ou de religion. Elle respecte toutes les croyances (...) »; Art. 1 de la loi du 9 décembre 1905: "La République assure la liberté de conscience. Elle garantit le libre exercice des cultes sous les seules restrictions édictées ci-après dans l'intérêt de l'ordre public. »
} 
considérer qu'il est, du seul fait de l'existence de règles appropriées en ce domaine, satisfait à l'exigence de liberté religieuse ${ }^{26}$. La pleine revendication du principe de liberté de religion s'avère incontestablement restreinte par le rôle prédominant accordé en France au principe de laïcité ${ }^{27}$, avec pour conséquence que la garantie de la liberté de religion semble en quelque sorte déconnectée du bloc de dispositions qui vont par ailleurs gérer les organisations et activités cultuelles.

\section{Un droit souple et adaptable}

Le droit français des religions serait ainsi d'un accès difficile, morcelé, peu lisible, centré sur la gestion des organisations et déconnecté des principes fondamentaux... Le rôle occupé par les circulaires et la pratique administrative lui confère en outre un caractère précaire, vecteur d'une certaine incertitude juridique. Ces faiblesses ont cependant pour contrepartie d'accorder à ce droit souplesse et adaptabilité. Un grand nombre de dispositions qui continuent d'être appliquées aujourd'hui sont antérieures aux années 1950, alors que le paysage confessionnel s'est depuis profondément diversifié. Elles ont été dans bien des cas modifiées au fil du temps ou ont fait l'objet d'aménagements par la pratique administrative et d'interprétations libérales par la jurisprudence, pour tenir compte notamment d'un contexte de pluralisme religieux. Ainsi, si le décret du 16 août $1901^{28}$ cite l'évêque et l'ordinaire du lieu à propos de demande d'autorisation d'une congrégation, le Conseil d'État saisi d'un projet de décret de reconnaissance d'une communauté bouddhiste a estimé en 1988 que «le texte de 1901 pouvait être interprété comme imposant d'une façon générale la production d'une attestation de la personnalité ayant qualité (...) pour exercer sur la communauté concernée un pouvoir juridictionnel comparable à celui de l'évêque sur les établissements existant dans son diocèse ; qu'en conséquence, il n'était pas nécessaire de modifier ou de compléter les textes législatifs ou réglementaires applicables aux congrégations religieuses pour en étendre le bénéfice à des communautés non catholiques ${ }^{29}$. En matière de sépultures, la loi du 14 novembre 1881 a supprimé la possibilité d'établir dans les cimetières des séparations entre lieux d'inhumation en fonction des différents cultes. Dès 1975 cependant, une circulaire $^{30}$ du ministère de l'Intérieur proposait aux maires d'autoriser les « regroupements de fait », pour apporter " une solution particulière au problème de l'inhumation de nos compatriotes musulmans ». La circulaire du 19 février $2008^{31}$ en vigueur aujourd'hui demande dans le même esprit aux préfets d'« encourager les maires à favoriser (...) l'existence d'espaces regroupant les défunts de même confession », en prenant soin de respecter le principe de neutralité du cimetière et la liberté de croyance individuelle. Des ajustements ont été apportés à la législation par le moyen de simples circulaires pour répondre aux demandes concrètes auxquelles sont confrontés les élus locaux. Plutôt que la voie de la réforme législative qui pourrait être source de controverse idéologique et politique, c'est celle d'un compromis pragmatique qui a été ici choisie, au risque de voir discutée la légalité des "carrés confessionnels " dont le statut juridique, et donc la pérennité, est en tout état de cause fragile. Dans les dernières décennies, c'est par de tels ajustements de fait qu'ont été résolues des demandes relatives aux dispenses d'assiduité scolaire, aux abattages rituels ou à la procédure de désignation des aumôniers hospitaliers ${ }^{32}$.

\section{Un droit qui reste à construire}

\footnotetext{
${ }^{26}$ FRANCE. CONSEIL D'ÉTAT, Un siècle de laïcité. Rapport public 2004, Paris, La Documentation française, 2004, p. 323-324.

${ }^{27}$ V. F. MESSNER, P.-H. PRÉLOT, J.-M. WOEHRLING (dir.), op. cit., nº 1091.

${ }^{28}$ Décret du 16 août 1901 modifié portant règlement d'administration publique pour la loi du $1^{\text {er }}$ juillet 1901 relative au contrat d'association, art. 19, 20 et 23.

${ }_{29}^{29}$ Question écrite $n^{\circ} 09532$ de M. Charles de Cuttoli : JO Sénat Q, 24 mars 1988, p. 412

${ }^{30}$ Circulaire $\mathrm{n}^{\circ} 75-603$ du 28 novembre 1975 - Inhumation des Français de confession islamique : BOI $\mathrm{n}^{\circ} 12 / 1975$

p. 275

31 Précitée.

${ }^{32}$ F. MESSNER, P.-H. PRÉLOT, J.-M. WOEHRLING (dir.), op. cit., nº 1198.
} 
L'adoption des deux lois du 15 mars 2004 et du 11 octobre 2010 a révélé que les questions liées à l'exercice du culte et à sa visibilité étaient source de tension et de débats que ces dispositions prohibitives n'ont pas épuisés ${ }^{33}$. La voie législative a été le moyen d'affirmation d'une volonté politique de contrôle et d'expression de valeurs tout autant symboliques que juridiques, sans qu'ait pu être initiée cependant une véritable méthode de régulation des croyances religieuses dans l'espace public ou dans les relations interpersonnelles. Dans un climat de crispation face à des revendications fondées sur la religion, l'adaptation des textes par la pratique administrative ou le "management de proximité ${ }^{34}$ semble par ailleurs en panne dans un certain nombre de domaines, tels la construction d'édifices cultuels, le port de signes religieux ou l'expression religieuse sur le lieu de travail.

C'est sur cette question des manifestations de la liberté religieuse que notre conception d'un droit des religions centré sur des règles de gestion et d'organisation montre ses limites. L'enjeu n'est pas tant d'actualiser, de codifier ${ }^{35}$ ou même d'adapter ses dispositions pour répondre aux exigences nouvelles d'une société pluraliste, mais plutôt d'y réintroduire et de mettre en évidence la place prépondérante que doivent occuper les principes fondamentaux, et en premier lieu la liberté de religion, au sommet de ce corpus des textes de droit français des religions. En plus d'apporter lisibilité et stabilité, cette conception nouvelle de l'édifice normatif consoliderait les avancées de la jurisprudence et de la pratique administrative en matière de prise en compte de l'expression publique des religions ou de respect des prescriptions religieuses. Dialoguant avec d'autres concepts clés de notre ordre juridique (neutralité, égalité, ordre public...), elle contribuerait à faire de notre droit des cultes un véritable droit des religions.

\footnotetext{
${ }^{33}$ V. par exemple, en 2013 , les débats sur l'extension du principe de neutralité au secteur privé ou sur le port de signes religieux à l'université.

${ }^{34} \mathrm{~V}$. notamment les résultats de l'enquête de l'Institut Randstad et l'OFRE sur le fait religieux en entreprise, 27 mai 2013.

${ }^{35}$ V. J.-P. MACHELON (dir.), op. cit., p. 17.
} 